\title{
Design of Elevator Monitoring and Alarm System Based on WiMAX
}

$$
\text { Hong Jiang }{ }^{1, a} \text {, Yongfang Shi, }{ }^{2, b} \text {, Lei Qi }{ }^{1, c}
$$

${ }^{1}$ The School of Software, Xinjiang University, Xinjiang Urumqi 830008,China

${ }^{2}$ The School of Medical Engineering, Xinjiang Medical University, Xinjiang Urumqi 830011, China

aonlyxjjh@xju.edu.cn, bonlyxjsyf@126.com, c $254857301 @ q q . c o m$

\author{
Keywords: WiMAX, Monitoring and alarm system, Elevator
}

\begin{abstract}
With rapid speed, wide bandwidth and large covered range, WiMAX is used for building an system of elevator monitoring and alarm in this paper. The system integrates fault detection, trapped people communication and information management to inform maintenance staff. The design mentality and the structure of the system are detailed in the paper. The design provides a solution for reforming elevators by internet of things.
\end{abstract}

\section{Introduction}

With the gradual advancing of China's urbanization, the number of urban high-rise building continues to grow. The elevator is playing an indispensable conveyance in city vertical traffic system [1]. It is estimated that the elevator installations have exceeded 28 million in China by 2013, and the volume continues to rise. But, as the same time, safety accident in elevator happened frequently, which is causing wider concern in recent years. The reason is that the former manual operational maintenance services for elevators cannot accommodate the current growing number of aging elevators. So, it is urgent that an advanced and effective method must be adopted to safeguard the safe operation of the elevator. Along with the rapid development of IOT (Internet of Things) and the country's strong support, technologies for IOT are applied to various fields more and more widely [2]. As a typical application of IOT, elevator monitoring system can provide a more efficient management model. By using the elevator monitoring system with IOT, the elevator accidents could be predicted successfully and elevator failures could be repaired effectively so as to ensure the security of lifts and reduce the accident frequency rate and losses [3].

Recently, with the determination of IEEE 802.16e and IEEE $802.16 \mathrm{~m}$, WiMAX can provide transmission of video and audio signals based on IP[4], which makes up for the shortage of 3G and WLAN. So the wireless elevator monitoring based on WiMAX can meet the requirement of the transformation by the Internet of Thing.

\section{Introduce of elevator monitoring alarm system}

Elevator monitoring alarm system is failure judgment and warning system for elevator, which is based on elevator detection technology. Early elevator detection system belongs to a part of elevator running control system, which is used to improve elevator's stability by feeding the real-time data to control terminal. These real-time data mainly rely on sensors which are distributed in elevator to gather various parameters of running elevator such as the weight of the lift car, the signal of portal crane, the signal of the layer precision of the elevator, the signal of safety gear and so on. Now the elevator detection system has gradually improved because of the need for elevator fault diagnosis. With the application of more advanced sensors, the real time running status of elevator can be sensed more detailedly and comprehensively. The elevator monitoring alarm system that made full use of advanced sensing technology and combined with modern communication technology can transfer the information from many elevators in a certain area to the monitor computer. The compute can analyze and judge the elevator failures. The elevator maintenance staff can deal with these failures according the feedback timely when the elevator is abnormal. 
WiMAX communication technology[5-6]. WiMAX(Worldwide Interoperability for Microwave Access)) which released by IEEE(Institute of Electrical and Electronics Engineers)is a standard of wireless broadband. Since 2001, there have been multiple releases(802.16 and $802.16 \mathrm{~m})$, the release of IEEE802.16e is adopted in elevator monitoring system. The standard can provide fixed broadband and mobile broadband access services, the effective frequency band is 2-6GHz and the duplex work form is FDD(Frequency Division Duplexing) and TDD(Time Division Duplexing). The accurate of invisible covers radius has reached Km level and the peak transmit rate can reach 75Mbit/s. The standard supports all-IP network-layer protocols and QoS(Quality of Service) technology, which meets requirements completely to building wireless elevator monitoring communication network in area.

The topology of monitoring and alarm system. Combining such advanced technologies as embedded computer technology, WiMAX wireless communication technology, elevator fault detection technology and IOT technology, this monitoring and alarm system join up the following elements including the end of elevator fault monitoring, residential property, maintenance company, local special equipment inspection institute and the computer of monitoring center to constitute the multi-level elevator monitoring and alarm network implements centralized management of elevator. $\mathrm{Bu}$ using this system, the elevator owner can real-time monitor operating condition of elevator and maintenance company can access the operational parameters of elevator to troubleshoot remotely, make plan and organize maintenance work. The local special equipment inspection institute can effectively supervise the operation of elevator according to the data of maintenance and operating conditions. The system can raise management level of elevator and the level of quality of maintenance. The topology of monitoring and alarm system is shown in Fig.1.

The elevator monitoring and alarm system includes elevator, monitoring terminals, WiMAX base station, local server, central server, monitoring PC and hand-held intelligent terminals, the function of every part is described below:

The monitoring terminal that lays bottom layer of system is very important part, in which a set of sensor is installed and typical failure reference point is set. The sensor is responsible for collecting operational parameters of elevator. In the normal operation conditions, the elevator can be accessed locally and remotely. But when abnormality of running elevator is raised, a warning will be triggered automatically or manually and a transport channel of voice and video will be set up on request. Meanwhile, the parameters of failure status are uploaded. WiMAX base station connects local servers in small residential areas, by which many work can be carry out effectively such as building broadband wireless network, allocating network identity for each terminal, accepting request for network access from terminal, receiving the uploaded data from each terminal and transiting the data to local servers. The local server is responsible for counting, collating the parameters of each elevator in area and storing them to the database. Local and remote queries can be allowed and the local database synchronizes with the server database of local special equipment inspection institute on a regular schedule by internet.

Hardware constitution of monitoring and alarm terminals. The monitoring and alarm terminals of elevator are key components of fore-end hardware in system, which include embedded ARM computer, data collection module, wireless communication module, control module, multimedia module and power module. The structure of elevator monitoring and alarm terminal is shown in Fig. 2. 


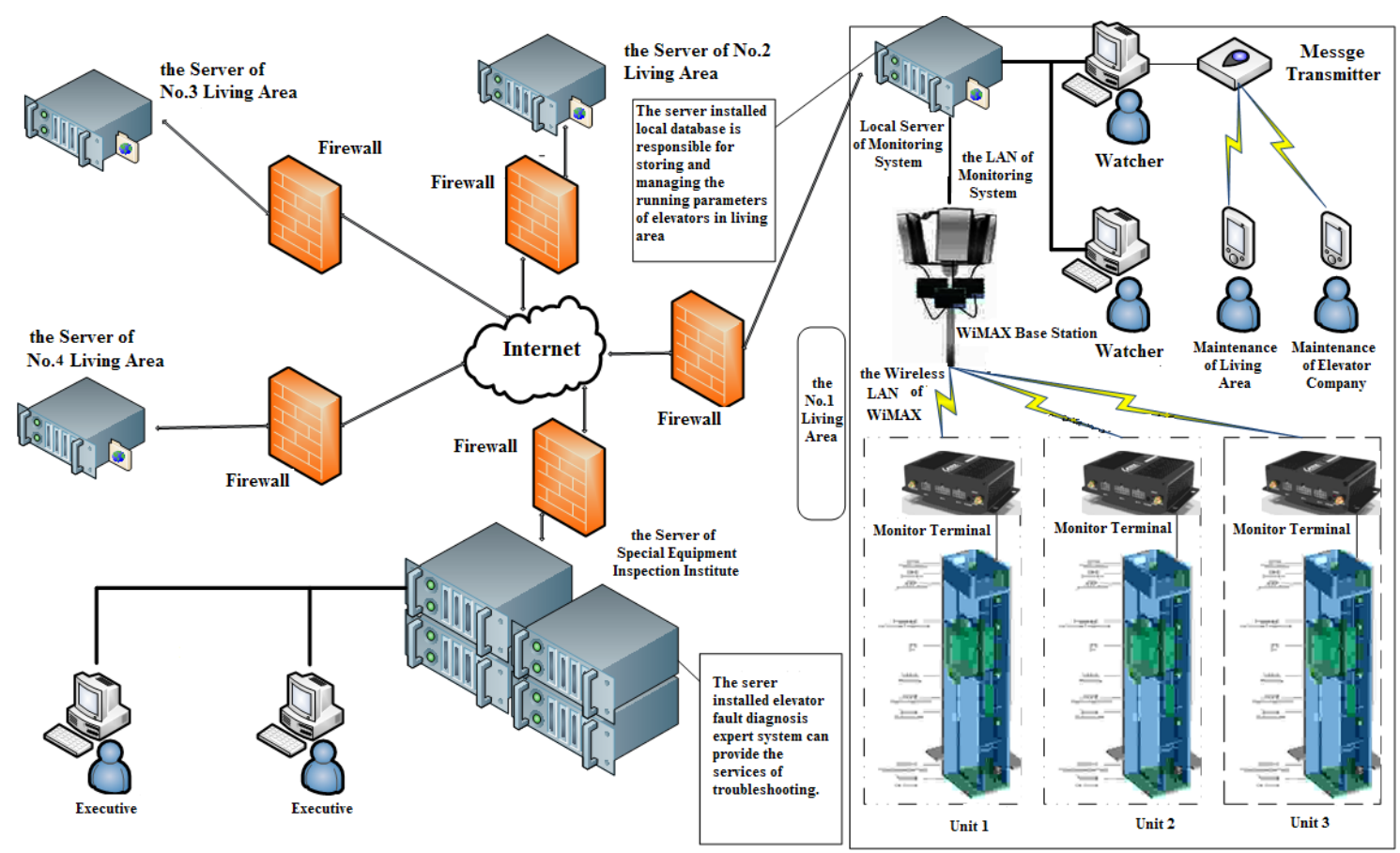

Fig.1 the Network topology of wireless elevator monitoring alarm system

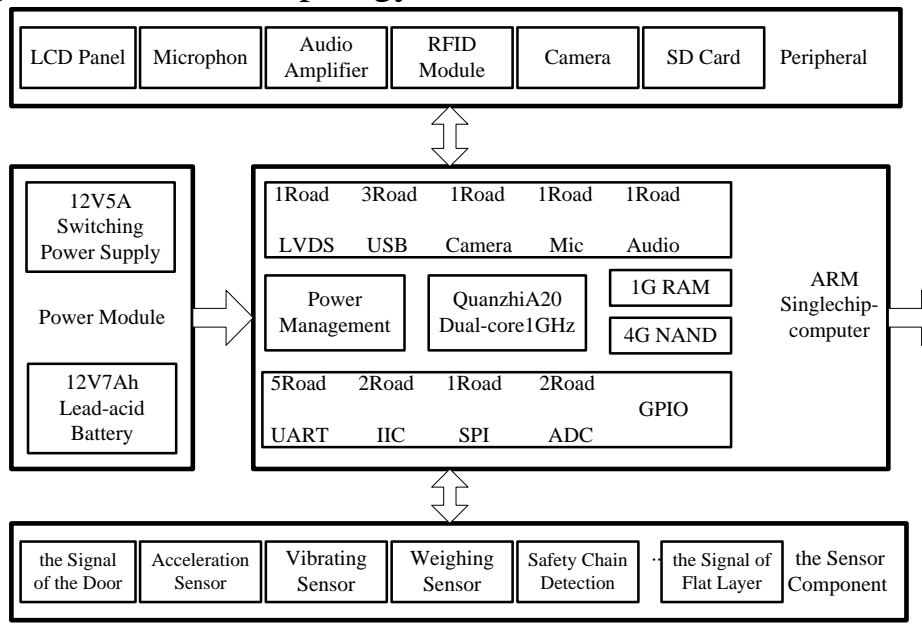

Fig.2 the Structure of elevator monitoring alarm terminal

The master computer. The master computer adopt industry single-board controller in which Quanzhi A20 chip is kernel component. CPU---ARM Cortex A7---is a dual 32-bit low power microprocessor, which onboard peripheral hardware resources include 1GB memory, 4GB NAND Flash, 5 full duplex serial ports, 2 IIC interfaces, 1 SPI interface, 2 10-bit ADC conversion interfaces and multi common $\mathrm{I} / \mathrm{O}$ interfaces for connecting various sensors and wireless communication module.

Collection module. The collection module is composed of multiple sensors allocated in all parts of elevator. It should be noted that the sensors are independent of the elevator control system and mainly used for collecting operational parameters of key components of elevators in real time. The kinds of these sensors include accelerometer, load cell, vibration sensor, leveling sensor and door motor sensor, which are connected to monitoring and alarm terminals by signal cable.

Multimedia module. The multimedia module consists of 14-inch LCD, audio power amplifier, loudspeaker, electret microphone and camera. When the elevator is normally running, multimedia can play propaganda about taking lift-related knowledge or multi-media advertisement. The cost of operation and maintenance of system can be offset by advertisement revenue. When the elevators have faults, the multimedia module by which the audio and video signals inside the elevator are collected and transferred can be used as a rescuing and alarm virtual phone for trapped persons. 
Power module. The power module is consisted of the main power and backup power. The main power is a switching power supply whose input and output is AC150-220V and DC12V5A respectively. The backup power is an uninterrupted power supply which is composed of 12V7AH lead-acid batteries and charge and discharge managing circuit for guaranteeing continued operating for at least one hour after power-off.

Communication module. The communications network of elevator monitoring and alarm system is built using WiMAX wireless broadband technologies. The communication module is realized by Intel Centrino Advanced-N + WiMAX6250 wireless card conforming IEEE802.16e Wave 2 protocol. Some basic parameters of card are described as: 3.5G working frequency band, 6Mbps uplink bandwidth, 20Mbps downlink bandwidth, Mini PCIe half-high design and USB 2.0 communication port. Intel provides official drivers of Linux and Windows.

I/O module of radio frequency identification (RFID). The operations people and managers of elevator are equipped identification card which are using for recording the log about the start and end time of maintenance works, identity data of operations staff. This module adopts RFID technology and use Mingtai MRF-35-MEM as the contactless IC card reader. The type of reading and writing IC card meets the criteria of MIFARE, the frequency of reading and writing is $13.56 \mathrm{MHz}$, communication interface is RS232, communication frequency is $115200 \mathrm{bps}$.

WiMAX wireless network building. The IEEE802.16 standard designates two standard networking modes: PMP(point-to-multi-point) and $\mathrm{Mesh}^{[7]}$. Because the requirement of coverage radius of base station is not big, the amount of network terminal is small and the type of terminal is fixed, PMP mode is adopted. The complete network architecture of WiMAX is composed of core network, base station, user base station, radio relays and user terminal equipment. In the application environment of this system, the requirement of coverage radius of signals is about $1 \mathrm{~km}$, which is far lower than the standard --50km.

\section{The system software}

The system software includes two parts: terminal software and monitoring computer software, programming are used OS (operating system) +APP (application) mode, the terminal of OS using the Linux operating system to remove the graphical interface, the APP software based on the cross platform C++ application framework QT development. The monitoring uses Windows 8 and the APP is developed by Delphi based on Windows visual programming environment.

The terminal application program. According to the actual function, the terminal application program can be divided into four main modules: acquisition and processing, communication, event judgment processing and multimedia play.

Data acquisition module mainly collects running status parameters for the elevator. In order to ensure the reliability and real-time of data transmission, data transmission uses the TCP protocol, and the design of video data uses the UDP on the audio transmission based on RTCP protocol. The event judgment processing mainly analyzes sensor data and compares to the normal parameters. According to the data types, if fault type is determined it will trigger the alarm and upload the fault parameters, in addition to accept the execution of remote access and command parsing. Multimedia playing module is responsible for audio, video file decoding and playing.

The monitoring computer application. According to the function, the monitoring computer applications can be divided into communication, data processing, transaction processing, man-machine interface of four modules.

Communication module includes four parts: 1 , receiving terminal data package uploaded through TCP protocol, sending instruction to realize communication between the remote monitoring computer and terminal interaction. 2, through the RTCP protocol to receive video telephone terminal data flow, and publish the update advertisement file. 3, If emergency is happened, expert consultation can be established to realize multi voice communication through IP phone. 4, the necessary elevator status can be sent to rescue or maintenance personnel mobile phone via text message. 


\section{Conclusions}

(1) WiMAX, as a metropolitan area network technology based on open standards, can provide wireless broadband connections in the wider geographical range compared with the WIFI technology.

(2) There is nothing comparable to this advantage in the non visible distance up to the Km range for requirement to establish the high bandwidth of the wireless local area network with wireless occasions.

(3) The technology to build the alarm system is more convenient for installation layout, flexibility and maintainability.

(4) It provides a solution for the present stage of IOT transformation to use elevators aging in later life.

\section{Acknowledgements}

This work was financially supported by:

[1] Major Project of Autonomous Regions Colleges Scientific Research Plan in Xinjiang[XJEDU2014I007]

[2] The Joint Project of School and University of Xinjiang [201204361319]

\section{References}

[1] G.C. Zhang, J. Wang. Urban Research. 2001,(05):51-53. “In Chinese”

[2] Y. Peng, C.S. Zhou. Machinery Design \& Manufacture. 2013,(01):14-16. “In Chinese”

[3] Q.L. Huang, W. Yang and G.Xiao. Manufacturing Automation. 2013,(02):36-39. “ In Chinese”

[4] F. Zhang. Computer and Modernization. 2013(02):210-212. "In Chinese”

[5] T. Tian, X.C. Zhang and X.J. Zhou. WiMAX Wireless Network Technology and Application. Beijing: Post \& Telecom Press,2009. “In Chinese”

[6] http://resources.wimaxforum.org/resources/documents/marketing/whitepapers

[7] IEEE Std802.16e.IEEE Standard for Local and Metropolitan Area Networks-part 16:Air Interface for Fixed Broadband Wireless Access Systems. 2005(12).W.F. Wu. 